\title{
3-Dimensional analysis of holographic memories based on photopolymers using finite differences method
}

\author{
Sergi Gallego ${ }^{1}$, Manuel F. Ortuño ${ }^{1}$, Cristian Neipp ${ }^{1}$, Andrés Márquez, Augusto Beléndez ${ }^{1}$, Elena \\ Fernández ${ }^{2}$, John V. Kelly ${ }^{3}$, and John T. Sheridan ${ }^{3}$. \\ ${ }^{1}$ Dept. Física Enginyeria de Sistemes i Teoria del Senyal, Universitat d'Alacant (Spain) Apartat 99 \\ E-03080 Alacant \\ ${ }^{2}$ Dept. Interuniversitari d'Òptica, Universitat d'Alacant (Spain) Apartat 99 E-03080 Alacant \\ ${ }^{3}$ Dept. of Electronic and Electrical Engineering, University College Dublin, Belfield, Dublin 4, \\ Republic of Ireland
}

\begin{abstract}
The characterization of the behavior of photopolymers is an important fact in order to control the holographic memories based on photopolymers. In recent years many 2-dimensional models have been proposed for the analysis of photopolymers. These models suppose that the photopolymer layer is homogeneous in depth and good agreement between theoretical simulations and experimental results has been obtained for layers thinner than $200 \mu \mathrm{m}$. The attenuation of the light inside the material by Beer's law is an important factor when higher thickness are considered. In this work we use a Finite-Difference method to solve the 3 dimensional problem. Now diffusion in depth direction and the attenuation of the light inside the material by Beer's law are also considered, the influence of the diffusivity of material in the attenuation of the refractive index profile in depth is analyzed.
\end{abstract}

Keywords: Holography, Photopolymers, Data Storage, Holographic recording materials

\section{INTRODUCTION}

The versatility of photopolymers in optics has been shown by many authors [1-3]. Their good optical properties, low cost and easy way in which their chemical composition may be changed, thickness and other material parameters make new applications for the use of photopolymers possible. In optics, one of the most interesting propositions is to design holographic memories based on photopolymers. In recent years, research to achieve optimized holographic memories has received much attention [4-8]. One of the basic requirements for holographic memories to be competitive is that the thickness of the layer of recording material must be $500 \mu \mathrm{m}$ or more [3]. A greater number of holograms may be recorded with thicker layers, since in this case the angular Bragg selectivity is higher and the width of the angular response curve is very small [3]. Many materials have been employed as holographic memories (e.g., inorganic crystals, bacteriorhodopsin, photopolymers) [3,9]. Recently, new companies (Aprilis, Inphase, etc) have used photopolymer disks to optimize the first commercial holographic memories. The Aprilis HMD company employed a photopolymer with a storage density greater than $100 \mathrm{bits} / \mu \mathrm{m}^{2}$ and dynamic range $(\mathrm{M} / \#)$ around 22 [4], and the Inphase Group presented a photopolymer with stringent criteria for dynamic range, photosensitivity, non-destructive readout and thermal stability [5].

Layers of photopolymer about $1 \mathrm{~mm}$ thick allow many diffraction gratings to be recorded in the same volume of photopolymer using different multiplexing techniques. This has great practical importance in the field of WORM (write once read many) type holographic memories. Therefore, characterization of the behavior of photopolymers is important in order to control the holographic memories based on these compounds. In recent years many 2-dimensional models have been proposed for the analysis of photopolymers [10-16]. Many parameters have been introduced to theoretically control the photopolymer behavior. In the 1990's the first models that took into account the importance of the polymerization rate and monomer diffusion processes in the description of hologram formation appeared. In the work of Adhami et al. [10] equations governing the formation of the hologram were first proposed and solved. Zhao and Moroulis proposed a different model [11], where the monomer diffusion assumes quite an important role when a sinusoidal exposure is used. In their model one assumes a linear relation between polymerization and incident intensity, in addition a linear relation between index modulation and polymer concentration is postulated. The differential equations provide a semi-analytical way of explaining why the index modulation is smaller when the separation between strips increases, that is to say, when low space frequencies are recorded. Subsequently, a lot of new models

Photon Management II, edited by John T. Sheridan, Frank Wyrowski, Proc. of SPIE Vol. 6187,

618714, (2006) -0277-786X/06/\$15 - doi: 10.1117/12.662481

Proc. of SPIE Vol. 6187 618714-1 
were presented, taking into account different aspects of the hologram formation process [12-16]. Some of the most interesting novelties were introduced by Sheridan and co-workers, specifically, introduction of the non-local polymerization concept and the high spatial frequency cut-off [14]. On the other hand, a new model developed by Southerland analyzes the effects of shrinking and swelling [16], and is an interesting extension to the non-local polymerization-driven diffusion model. All of these models assume that the polymer and monomer concentration can be described using two or four harmonics in polymer and monomer concentration. Nevertheless, recently, a way to solve the photopolymer behaviour was introduced by $\mathrm{Wu}$ et al. [17], thus the Finite-Difference method was used to solve the grating formation in photopolymers in the non-local space response case, and J. V. Kelly et al [18] solved the equations for non-local temporal response using the Finite-Difference method too. But all of these models assume that the photopolymer layer is homogeneous in depth and good agreement between theoretical simulations and experimental results has been obtained for layers thinner than $200 \mu \mathrm{m}$. The attenuation of light inside the material according to Beer's law results in an attenuation of the index profile inside the material and in some cases the effective optical thickness of the material is lower than the physical thickness [19]. Some approximations have been made in order to understand the profile recorded in thick photopolymers [20]. In this study we use a Finite-Difference method to solve the 3 dimensional local problem. This method has been applied successfully to thin layers [17-18]. Now diffusion in depth and the attenuation of light inside the material according to Beer's law are also considered, and the influence of the diffusivity of the material on attenuation of the refractive index profile in depth is analyzed.

The intensity distribution is assumed constant along $y$, but attenuated in depth along the $Z$ axis. The depth attenuation changes the angular response of the grating stored in the material [21] and determines the optical thickness and ultimate storage capacity of each material composition [19-20]. Assuming Beer's law explains the attenuation of light intensity with depth, the recording intensity can be written as:

$$
I(x, z, t)=I_{0}\left[1+V \cos \left(K_{g} x\right)\right] e^{-\alpha(t) z}
$$

where $K_{g}$ is the grating number of the recorded grating, which depends on the grating period and may be written as:

$$
K_{g}=\frac{2 \pi}{\Lambda}
$$

In this paper we analyzed gratings with 1125 lines $/ \mathrm{mm}$ ( $\Lambda$ is around $0.88 \mu \mathrm{m}$ in this work).

$\alpha$ is the coefficient of light attenuation. The initial value of $\alpha\left[\alpha(t=0)=\alpha_{0}\right]$ can be obtained if the transmittance and the physical thickness of the layer are known. In our material [20] for layers with a physical thickness around $800 \mu \mathrm{m}$, the transmittance is around $0.82 \%$; in others words, the values of $\alpha_{0}$ for this composition are around $0.006 \mu \mathrm{m}^{-1}$ [20]. The absorption of the layer changes as a function of the time it takes for the dye to be consumed. In a first approximation, it can be written as:

$$
\alpha(\mathrm{t})=\alpha_{0} \mathrm{e}^{-\mathrm{K}_{\alpha} \mathrm{I}_{0}{ }^{\beta} \mathrm{t}}
$$

where $\beta$ is a constant that determines the influence on the intensity as the dye is consumed and depends on many factors (chemical composition of the material, temperature, humidity, etc.). The absorption decay depends on the dye and the intensity used, and $K_{\alpha}$ defines the rate at which the dye is consumed. For a power intensity of $5 \mathrm{~mW} / \mathrm{cm}^{2}$ and $\beta=0.5$, the constant takes the following value: $K_{\alpha}=0.005 \mathrm{~cm}^{2} \mathrm{~mW}^{-1} \mathrm{~s}^{-1}$ for an initial approximation (see reference 20).

\section{THEORETICAL ANALYSIS}

We propose use the following two equations that governs the 3-dimensional polymerization:

$$
\begin{gathered}
\frac{\partial[\mathrm{M}](\mathrm{x}, \mathrm{z}, \mathrm{t})}{\partial \mathrm{t}}=\frac{\partial}{\partial \mathrm{x}} \mathrm{D} \frac{\partial[\mathrm{M}](\mathrm{x}, \mathrm{z}, \mathrm{t})}{\partial \mathrm{x}}-\mathrm{k}_{\mathrm{R}}(\mathrm{t}) \mathrm{I}^{\gamma}(\mathrm{x}, \mathrm{z}, \mathrm{t})[\mathrm{M}](\mathrm{x}, \mathrm{z}, \mathrm{t})+\frac{\partial}{\partial \mathrm{z}} \mathrm{D} \frac{\partial[\mathrm{M}](\mathrm{x}, \mathrm{z}, \mathrm{t})}{\partial \mathrm{z}}, \\
\frac{\partial[\mathrm{P}](\mathrm{x}, \mathrm{z}, \mathrm{t})}{\partial \mathrm{t}}=\mathrm{k}_{\mathrm{R}}(\mathrm{t}) \mathrm{I}^{\gamma}(\mathrm{x}, \mathrm{z}, \mathrm{t})[\mathrm{M}](\mathrm{x}, \mathrm{z}, \mathrm{t})
\end{gathered}
$$


where $n$ is the refractive index, $[M]$ is the monomer concentration, $[P]$ is the polymer concentration, and $\gamma$ indicates the relationship between intensity and polymerization rate $\left(k_{R}\right)$. In this paper, we assume $\gamma=0.5-\mathrm{a}$ non-linear intensity response.

We assume that the polymerization rate decreases due to the Trommsdorff effect [20], and is given by:

$$
k_{R}(t)=k_{R} \exp \left(-\varphi I_{0} t\right)
$$

where $\varphi$ is the attenuation coefficient of the polymerization rate.

In previous studies some approximations have been made in order to solve the differential equations [20]. In this paper we use the Finite-Difference method to solve a 3-dimensional problem using a rigorous method. The first step to find the numerical solutions is to transform the monomer and polymer concentration into a matrix. $M(x, y, z)$ can be rewritten as $M(x, z)$ because it is constant along the y axis. In this work we defined the size of the matrix as 1000x1000. When the initial conditions are defined we use the discretized diffusion equations and Taylor series expansion of $M(x, z, t)$ around a given point $(x, z)$. Therefore we can obtain the value of $M(x, z, t)$ and $P(x, z, t)$ as the elements $M_{i, j, k}$ and $P_{i, j, k} . M_{i, j}$ is the monomer concentration at the $i$ th and $j$ th dimension space point and $\Delta x$ and $\Delta z$ are the space increments in the $\mathrm{X}$ and $\mathrm{Z}$ directions. Then we present the Taylor expansion for the $x$ component, and the expansion for the $z$ component is similar.

$$
\frac{\partial M_{i, j, k}}{\partial x_{i, j, k}} \cong \frac{1}{2 \Delta x}\left(M_{i+1, j, k}-M_{i-1, j, k}\right)
$$

and

$$
\frac{\partial^{2} M_{i, j, k}}{\partial x^{2}{ }_{i, j, k}} \cong \frac{1}{\Delta x^{2}}\left(M_{i+1, j, k}-M_{i, j, k}+M_{i-1, j, k}\right),
$$

We discretize the diffusion equation in the time domain using again a Taylor series expansion around a given time $t$ :

$$
\frac{\partial M_{i, j, k}}{\partial t_{i, j, k}} \cong \frac{1}{\Delta t}\left(M_{i, j, k+1}-M_{i-1, j, k}\right),
$$

where $M_{j}$ is the monomer concentration at the $j$ th time step and $\Delta t_{D}$ is the time increment. We can rewrite the intensity distribution inside the material in a discretized form:

$$
I_{i, j, k}=I_{0} e^{-\alpha j \Delta z(k)}\left[1+\cos \left(K_{g} i \Delta x\right)\right]
$$

Substituting Eqs. (7), (8) and (9) in Eq. (4) we can write the monomer diffusion expression as:

$$
\begin{aligned}
& \frac{M_{i, j, k+1}-M_{i, j, k}}{\Delta t}=D \frac{M_{i+1, j, k}-2 M_{i, j, k}+M_{i-1, j, k}}{\Delta x^{2}}+D \frac{M_{i, j+1, k}-2 M_{i, j, k}+M_{i, j-1, k}}{\Delta z^{2}} \\
& -k_{i, j, k} I_{i, j, k} M_{i, j, k}
\end{aligned}
$$

and expression for monomer concentration as: 


$$
\begin{aligned}
& M_{i, j, k}=\frac{\Delta t}{\Delta x^{2}} D M_{i+1, j, k-1}-2 \frac{\Delta t}{\Delta x^{2}} D M_{i, j, k-1}+\frac{\Delta t}{\Delta x^{2}} D M_{i-1, j, k-1}+\frac{\Delta t}{\Delta z^{2}} D M_{i, j+1, k-1} \\
& -2 \frac{\Delta t}{\Delta z^{2}} D M_{i, j, k-1}+\frac{\Delta t}{\Delta z^{2}} D M_{i, j-1, k-1}-\Delta t k_{i, j, k-1} I_{i, j, k-1} M_{i, j, k-1}+M_{i, j, k-1}
\end{aligned}
$$

Substituting Eqs. (7), (8) and (9) in Eq. (5) we can write the polymer formation expression as:

$$
\frac{P_{i, j, k+1}-P_{i, j, k}}{\Delta t}=k_{i, j, k} I_{i, j, k} M_{i, j, k}
$$

and the expression for polymer formation as:

$$
P_{i, j, k}=\Delta t k_{i, j, k-1} I_{i, j, k-1} M_{i, j, k-1}+P_{i, j, k-1}
$$

In order to study the numerical stability of the equations, the increment in the time domain, $\Delta t$, must satisfy the stability criterion [17-18]:

$$
\Delta t \leq \frac{1}{2} \frac{(\Delta x)^{2}}{D}
$$

In this paper we choose $\Delta t=0.4\left(\Delta x^{2} / D\right)$, which is consistent with the $\mathrm{Wu}$ and Glytsis [17] and J. V. Kelly dimensionless analysis [18]. However the diffusion model now includes diffusion in the $\mathrm{z}$ direction.

One of the problems associated with Finite-Difference codes is that the grid used for the simulations has a finite size. This implies that in order to simulate open regions some artificial tricks must be introduced when electromagnetic simulations are calculated using this method [22]. Now for polymer diffusion we want to find a suitable boundary condition to simulate the extension to large $(1 \mathrm{~cm}$ in diameter) real gratings recorded on the outer perimeter of the grid. There are 2 types of boundaries in our problem.:

The first is the boundaries of the polymer with other different media (the glass substrate and air). In this case we assume the monomer diffusion is only permitted in one direction. Therefore for the boundary condition between medium 1 (air) and medium 2 (polymer) of the grid, the monomer concentration (Equation 12) can be written as:

$$
\begin{aligned}
& M_{i, 1, k}=\frac{\Delta t}{\Delta x^{2}} D M_{i+1,1, k-1}-2 \frac{\Delta t}{\Delta x^{2}} D M_{i, 1, k-1}+\frac{\Delta t}{\Delta x^{2}} D M_{i-1,1, k-1}+\frac{\Delta t}{\Delta z^{2}} D M_{i, 2, k-1} \\
& -2 \frac{\Delta t}{\Delta z^{2}} D M_{i, 1, k-1}-\Delta t k_{i, 1, k-1} I_{i, 1, k-1} M_{i, 1, k-1}+M_{i, 1, k-1}
\end{aligned}
$$

And for the boundary between medium 2 (polymer) and medium 3 (glass substrate):

$$
\begin{aligned}
& M_{i, j \max , k}=\frac{\Delta t}{\Delta x^{2}} D M_{i+1, j \max , k-1}-2 \frac{\Delta t}{\Delta x^{2}} D M_{i, j \max , k-1}+\frac{\Delta t}{\Delta x^{2}} D M_{i-1, j \max , k-1} \\
& -2 \frac{\Delta t}{\Delta z^{2}} D M_{i, j \max , k-1}+\frac{\Delta t}{\Delta z^{2}} D M_{i, j-1 \max , k-1}-\Delta t k_{i, j \max , k-1} I_{i, j, k-1} M_{i, j \max , k-1}+M_{i, j \max , k-1}
\end{aligned}
$$

where $j_{\max }$ is the maximum value of $j$

The second is the boundary of the recorded zone. In this area we have non-exposed photopolymer, where we assume that the monomer concentration is constant and takes the value $M_{0}$, the initial monomer concentration. In the following equation $i=1$ ( the case for $i=i_{\max }$ is similar). 


$$
\begin{aligned}
& M_{1, j, k}=\frac{\Delta t}{\Delta x^{2}} D M_{2, j, k-1}-2 \frac{\Delta t}{\Delta x^{2}} D M_{1, j, k-1}+\frac{\Delta t}{\Delta x^{2}} D M_{0}+\frac{\Delta t}{\Delta z^{2}} D M_{1, j+1, k-1} \\
& -2 \frac{\Delta t}{\Delta z^{2}} D M_{1, j, k-1}+\frac{\Delta t}{\Delta z^{2}} D M_{1, j-1, k-1}-\Delta t k_{1, j, k-1} I_{1, j, k-1} M_{1, j, k-1}+M_{1, j, k-1}
\end{aligned}
$$

To minimize the effect of this boundary in the simulations we assume that the recording intensity can be rewritten as:

$$
I_{i, j}=I_{0} e^{-\alpha j \Delta z}\left[1+\cos \left(K_{g} i \Delta x+\pi\right)\right]
$$

Consequently if we simulate some exact grating periods: $I_{1, j} \approx I_{i \max , j}=0$.

This is an important drawback because the simulated gratings have a finite size, which is very small compared with the real grating $\left(1 \mathrm{~cm}\right.$ diameter around $10^{4}$ periods recorded $)$. Then, the boundaries generate a perturbation traveling to the center of the grid. The velocity of the perturbation depends on the monomer diffusion $(D)$ as calculated in reference [23], where the displacement $x$ of the diffusing species at time $t$ is given as:

$$
\bar{x}=2 \sqrt{D t}
$$

Some estimations of the monomer diffusivity in our photopolymeric material situate the value of $D$ below $10^{-10} \mathrm{~cm}^{2} \mathrm{~s}^{-1}$ and the exposure time is less than $10 \mathrm{~s}$. Using these parameters we obtain a displacement over a distance of $1.8 \mu \mathrm{m}$. Then in order to apply the boundary conditions and eliminate the boundary effects in the middle of the simulated grating, we consider 10 periods and assume that the central period is not affected by boundary perturbations.

One of the problems involved in working theoretically with thick layers (where the refractive index profile is attenuated in depth) is calculating the diffraction efficiency around the Bragg angle. The time evolution of the harmonic components of the polymer and monomer grating profiles is found by applying the discrete Fourier transform to the resulting data at each time step. Then we can obtain the refractive index modulation as a function of monomer and polymer gratings [24]. It is common in holography to express the profile recorded in the grating in terms of the refractive index. For this study we assume that a refractive index profile stored in the layer $n(z)$ has the form[25]:

$$
n(z)=n_{0}+n_{1}(z) \cos \left[K_{g} x\right]+n_{2}(z) \cos \left[2 K_{g} x\right]+n_{3}(z) \cos \left[3 K_{g} x\right]
$$

In our material $n_{2} \leq \frac{n_{1}}{8}$ and $n_{3}$ can be disregarded [25]. Therefore they do not affect the values of the higher harmonics in the diffraction efficiency around the first Bragg angle. In this paper we obtain the refractive index profile in each sub-layer using the diffusion model solved by the Finite Differences Method and we check if this method predicts agreement of the higher harmonics with the experimental measurements. We can relate the harmonic components of the refractive index to the Fourier components of the dielectric permittivity $(\varepsilon)$, provided that $\mathrm{n}_{1}<<\mathrm{n}_{0}$, in order to calculate an algorithm based on Rigorous Coupled Wave analysis (RCW) proposed by Moharam and Gaylord [27]. In this case:

$$
\varepsilon_{ \pm i}=n_{0} n_{i} \quad \text { for } \mathrm{i}=1,2,3
$$

Where $\varepsilon_{ \pm i}$ are the harmonics of the Fourier expansion of the dielectric permittivity.

The method used in this paper is described partially in previous papers $[19,21,26]$, but now we do not assume an exponential attenuation of the index in depth. In this paper the values of the refractive index profile are given by the model in each sub-grating. To apply the boundary conditions we assume, as in previous papers, that there are no reflected orders between the sub-layers inside the material.

\section{NUMERICAL SIMULATIONS}

In this section we present the first simulations using this method. We evaluate the importance of two specific aspects of monomer diffusion. The first is the importance of the higher harmonics in the recorded grating profile, and we analyze the behavior for different values of monomer diffusion. In the second part of the simulation the importance of monomer 
diffusion in depth in our material is studied. Then we can analyze the validity of the assumption made in previous studies where diffusion along the $\mathrm{z}$ axis was disregarded.

\subsection{Importance of diffusivity in the higher harmonics.}

Let us now study the influence of monomer diffusion on the index profile stored in the photopolymer. We used for our simulation the typical parameter values obtained in previous studies for $1 \mathrm{~mm}$ thick PVA/AA based photopolymer $\left(M_{0}=0.19 ; k_{R 0}=0.019 \mathrm{~cm}^{2} \mathrm{~mW}^{-1} \mathrm{~s}^{-1} ; \alpha_{0}=0.006 \mu \mathrm{m}^{-1}\right)$. The values of monomer diffusion in PVA/AA materials are around $4.10^{-11} \mathrm{~cm}^{2} \mathrm{~s}^{-1}$ [26]. This value represents the separation between viscous systems and liquid systems [12]. Viscous systems are characterized by the large values of the higher harmonics in the recorded grating. Simulations for monomer and polymer distribution for typical values of the PVA/AA system are shown in figure 1.

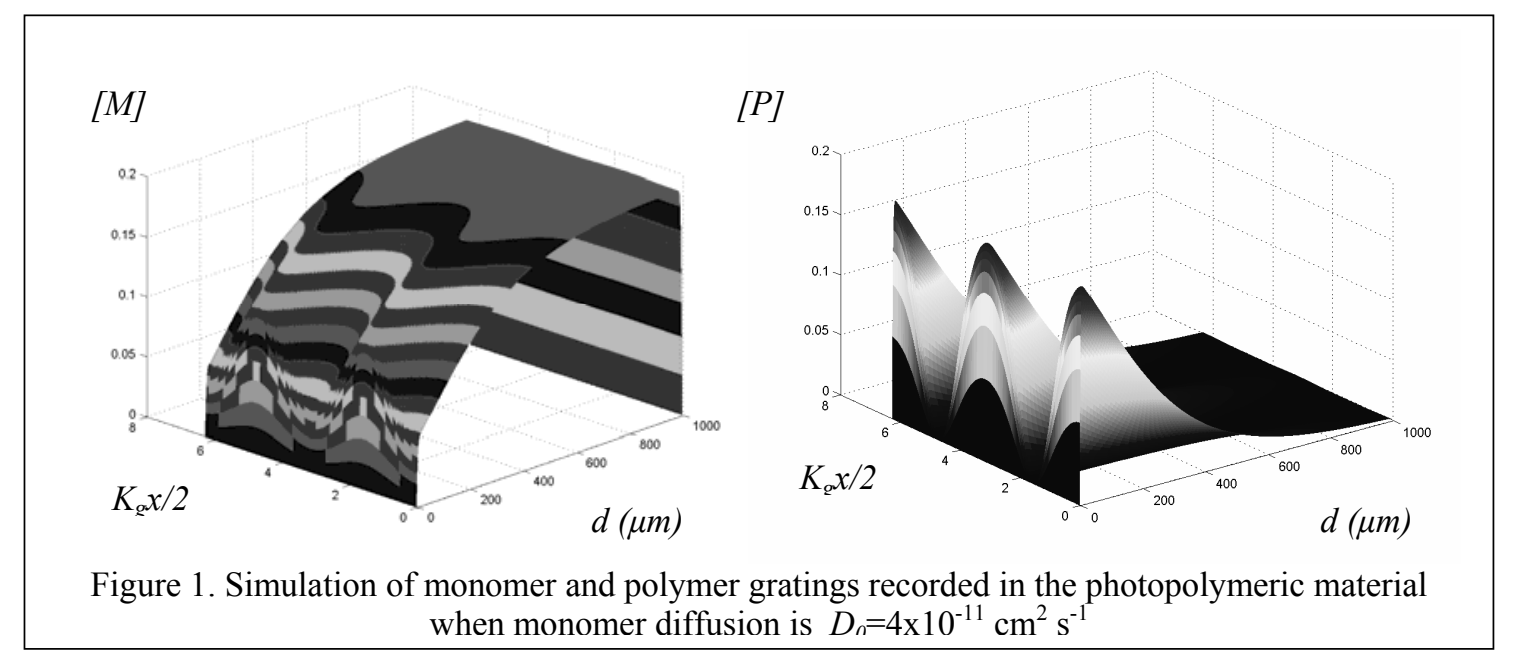

We can see the attenuation of the monomer and polymer profiles in depth according to Beer's law. On the other hand, we can see the residual monomer remaining in the last $400 \mu \mathrm{m}$ of the layer. It is interesting to evaluate the importance of the higher harmonics in the monomer and polymer profiles. These results are presented in Table I. The existence of third harmonics in the monomer concentration and forth harmonics in the polymer profile is demonstrated using different finite methods to solve the diffusion equations.

Table 1. Main harmonics of the monomer and polymer gratings $\left(D_{0}=4 \times 10^{-11} \mathrm{~cm}^{2} \mathrm{~s}^{-1}\right)$

\begin{tabular}{|l|l|l|l|l|l|l|l|}
\hline Depth & $\boldsymbol{M}_{\boldsymbol{0}}$ & $\boldsymbol{M}_{\boldsymbol{1}}$ & $\boldsymbol{M}_{\mathbf{2}}$ & $\boldsymbol{P}_{\boldsymbol{0}}$ & $\boldsymbol{P}_{\boldsymbol{1}}$ & $\boldsymbol{P}_{2}$ & $\boldsymbol{P}_{3}$ \\
\hline $\mathbf{1 0} \boldsymbol{\mu m}$ & 0.0858 & -0.0155 & 0.0007 & 0.1042 & 0.0456 & -0.0054 & 0.0003 \\
\hline $\mathbf{1 0 0} \boldsymbol{\mu m}$ & 0.1146 & -0.0134 & 0.0004 & 0.0754 & 0.0347 & -0.0025 & 0.0001 \\
\hline $\mathbf{5 0 0} \boldsymbol{\mu m}$ & 0.1779 & -0.0028 & 0.0000 & 0.0121 & 0.0060 & 0.0000 & 0.0000 \\
\hline
\end{tabular}

It is interesting to note that the values of the third harmonic of the monomer concentration $\left(M_{2}\right)$ and the fourth harmonic of the polymer concentration $\left(P_{3}\right)$ are very weak. Nevertheless the third harmonic of the polymer concentration cannot be neglected $\left(\left|P_{2}\right| \approx \frac{P_{1}}{9}\right.$ ). These results agree with the experimental measurements presented in previous papers.

Let us analyze more viscous photopolymeric systems. If we use the equations to simulate $D_{0}=10^{-12} \mathrm{~cm}^{2} \mathrm{~s}^{-1}$, we obtain monomer and polymer profiles like the gratings shown in figure 2. In this case we observe more modulation in the monomer grating (the second harmonic is three times higher than the previous case analyzed). We note that the monomer polymerizes before diffusion and the higher harmonics in the polymer are larger $\left(\left|P_{2}\right| \approx P_{1},\left|P_{3}\right| \approx \frac{P_{1}}{3}\right.$ and 
$\left|P_{4}\right| \approx \frac{P_{1}}{30}$, see Table 2). Therefore the secondary diffraction orders con not be disregarded. In previous papers we studied these diffraction orders in our material and based on these studies we determined that the monomer diffusivity in our material is higher than $10^{-12} \mathrm{~cm}^{2} \mathrm{~s}^{-1}$.

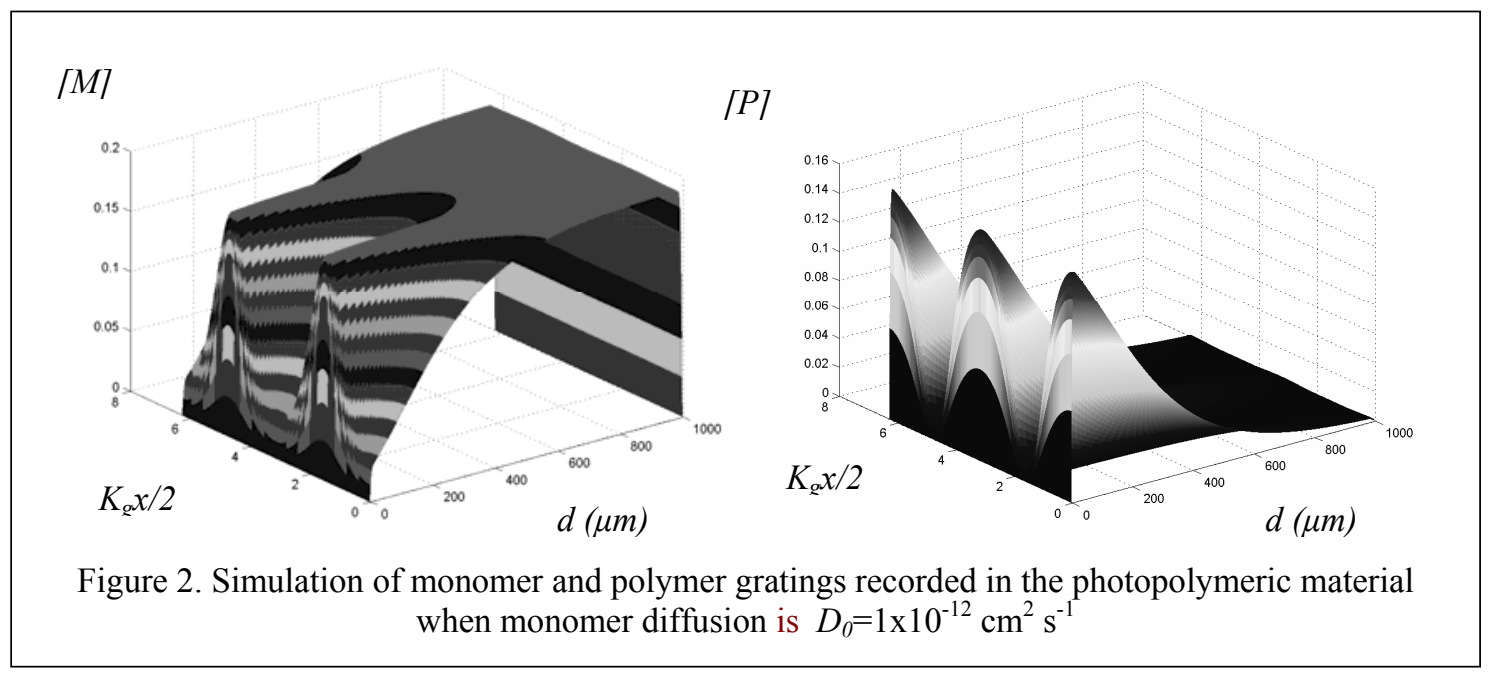

Table 2. Main harmonics of the monomer and polymer gratings $\left(D_{0}=10^{-12} \mathrm{~cm}^{2} \mathrm{~s}^{-1}\right)$

\begin{tabular}{|l|l|l|l|l|l|l|l|}
\hline Depth & $\boldsymbol{M}_{\boldsymbol{0}}$ & $\boldsymbol{M}_{\boldsymbol{1}}$ & $\boldsymbol{M}_{\mathbf{2}}$ & $\boldsymbol{P}_{\boldsymbol{0}}$ & $\boldsymbol{P}_{\boldsymbol{1}}$ & $\boldsymbol{P}_{\mathbf{2}}$ & $\boldsymbol{P}_{3}$ \\
\hline $\mathbf{1 0} \boldsymbol{\mu \mathbf { m }}$ & 0.0914 & -0.0376 & 0.0074 & 0.0986 & 0.0395 & -0.0353 & 0.0120 \\
\hline $\mathbf{1 0 0} \boldsymbol{\mu m}$ & 0.1237 & -0.0313 & 0.0035 & 0.0663 & 0.0324 & -0.0236 & 0.0118 \\
\hline $\mathbf{5 0 0} \boldsymbol{\mu m}$ & 0.1782 & -0.0042 & 0.0000 & 0.0118 & 0.0058 & -0.0001 & 0.0000 \\
\hline
\end{tabular}

To finalize this section we analyze more liquid polymeric systems, when the value of monomer diffusion is $D_{0}=10^{-10}$ $\mathrm{cm}^{2} \mathrm{~s}^{-1}$. We examine the 3 dimensional behavior of the polymeric system in figure 3 . As the value of $D_{0}$ increases, modulation of the monomer grating decreases and the shape of the polymer grating is more sinusoidal $\left(\left|P_{2}\right| \approx \frac{P_{1}}{15}\right.$, see Table 3). This illustrates the existence of a critical value of initial monomer diffusion in the evolution of the higher harmonics.
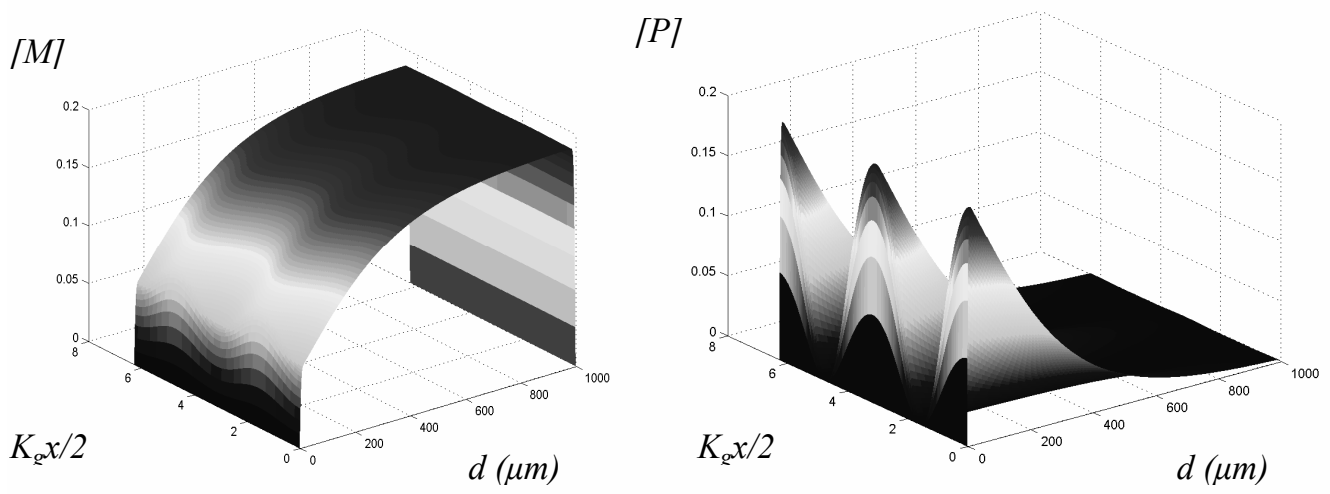

Figure 3. Simulation of monomer and polymer gratings recorded in the photopolymeric material when monomer diffusion is $D_{0}=1 \times 10^{-10} \mathrm{~cm}^{2} \mathrm{~s}^{-1}$ 
Table 3. Main harmonics of the monomer and polymer gratings $\left(D_{0}=10^{-10} \mathrm{~cm}^{2} \mathrm{~s}^{-1}\right)$

\begin{tabular}{|l|l|l|l|l|l|l|l|}
\hline Depth & $\boldsymbol{M}_{\boldsymbol{0}}$ & $\boldsymbol{M}_{\boldsymbol{1}}$ & $\boldsymbol{M}_{\mathbf{2}}$ & $\boldsymbol{P}_{\boldsymbol{0}}$ & $\boldsymbol{P}_{\boldsymbol{1}}$ & $\boldsymbol{P}_{\mathbf{2}}$ & $\boldsymbol{P}_{\mathbf{3}}$ \\
\hline $\mathbf{1 0} \boldsymbol{\mu \mathbf { m }}$ & 0.0818 & -0.0070 & 0.0001 & 0.1082 & 0.0501 & -0.0032 & 0.0001 \\
\hline $\mathbf{1 0 0} \boldsymbol{\mu \mathbf { m }}$ & 0.1126 & -0.0062 & 0.0000 & 0.0774 & 0.0369 & -0.0014 & 0.0001 \\
\hline $\mathbf{5 0 0} \boldsymbol{\mu \mathbf { m }}$ & 0.1778 & -0.0014 & 0.0000 & 0.0122 & 0.0060 & 0.0000 & 0.0000 \\
\hline
\end{tabular}

Based on the comparison of the three tables, there is a greater conversion of monomer into polymer for liquid systems and these systems present slightly less residual monomer at the end of the recording process. In the next section we investigate the effects of monomer diffusion in depth.

\subsection{Importance of the diffusivity in depth}

In a previous paper [20] it was assumed that monomer diffusion along the $Z$ axis is negligible compared with the importance of diffusion along the $X$ axis. Using the model presented in this study we can evaluate the real importance of diffusion along the $Z$ axis. Based on the above analysis, and assuming that all the monomer has not been consumed completely by the end of illumination, we could propose an accurate scheme to optimize the multiplexed holograms in this material. Now we simulate the grating formation for the same parameters in figure 3 , (where the monomer diffusion is more important) eliminating the diffusion in depth terms from equations 12 and 16-18. However, subsequent changes are shown in Table 4.

Table 4. Main harmonics of the monomer and polymer gratings without diffuision in $Z$ axis $\left(D_{0}=10^{-10} \mathrm{~cm}^{2} \mathrm{~s}^{-1}\right)$

\begin{tabular}{|l|l|l|l|l|l|l|l|}
\hline Depth & $\boldsymbol{M}_{\boldsymbol{0}}$ & $\boldsymbol{M}_{\boldsymbol{1}}$ & $\boldsymbol{M}_{\boldsymbol{2}}$ & $\boldsymbol{P}_{\boldsymbol{0}}$ & $\boldsymbol{P}_{\boldsymbol{1}}$ & $\boldsymbol{P}_{\boldsymbol{2}}$ & $\boldsymbol{P}_{\boldsymbol{3}}$ \\
\hline $\mathbf{1 0} \boldsymbol{\mu \mathbf { m }}$ & 0.0836 & -0.0071 & 0.0001 & 0.1064 & 0.0493 & -0.0032 & 0.0001 \\
\hline $\mathbf{1 0 0} \boldsymbol{\mu m}$ & 0.1126 & -0.0062 & 0.0000 & 0.0774 & 0.0369 & -0.0014 & 0.0001 \\
\hline $\mathbf{5 0 0} \boldsymbol{\mu m}$ & 0.1778 & -0.0014 & 0.0000 & 0.0122 & 0.0060 & 0.0000 & 0.0000 \\
\hline
\end{tabular}

Table 4 shows that the only appreciable changes are in the first $10 \mu \mathrm{m}$, but the variations of the harmonics is very small. These results agree with the average displacement after 10 seconds mentioned in the introduction $(1.8 \mu \mathrm{m})$. Therefore the assumption made in the previous paper (to disregard diffusion along the $Z$ axis) is valid for exposures around 10 seconds. Another question is the influence of diffusion along the $Z$ axis in the design of the multiplexed schedule where more than 1000 gratings are recorded - more than 1000 seconds are employed in the process.

\section{EXPERIMENTAL}

Obtaining the refractive index modulation as a function of the harmonics of the monomer and polymer gratings is well known. The refractive index modulation, $n_{l}(t)$, depends on the refractive indexes of the different components and following Aubrecht et al. [24], with a minor typographical correction (6 appears in the denominator of the first part of the right hand side of the equation instead of 3 , as has been shown in previous papers $[18,20,26]$ ) the refractive index modulation can be written as:

$$
n_{1}=\frac{\left(n_{\text {dark }}^{2}+2\right)^{2}}{6 n_{\text {dark }}}\left[-\left(\frac{n_{m}^{2}-1}{n_{m}^{2}+2}-\frac{n_{b}^{2}-1}{n_{b}^{2}+2}\right)[M]_{1}+\left(\frac{n_{p}^{2}-1}{n_{p}^{2}+2}-\frac{n_{b}^{2}-1}{n_{b}^{2}+2}\right)[P]_{1}\right]
$$

where: $n_{p}$, is the polymer refractive index, $\mathrm{n}_{\mathrm{m}}$, is the monomer refractive index, $n_{b}$, is the binder refractive index and $n_{\text {dark }}$, is the refractive index of the layer before exposure.

In the material used in this study the different refractive indexes take the following values: $n_{m}=1.486 ; n_{b}=1.474$; $n_{\text {dark }}=1.478 ; n_{p}=1.506$. These values were obtained using the Lorentz-Lorenz equation and the method used is described in references 20 and 22 . The calculations are based on refractometer measurements using water solutions.

Therefore we can simulate the distribution of the refractive index modulation in depth as plotted in figure 4 for $D_{0}=$ $4 \times 10^{-11} \mathrm{~cm}^{2} \mathrm{~s}^{-1,}$, where we can see the attenuation of the index profile. 


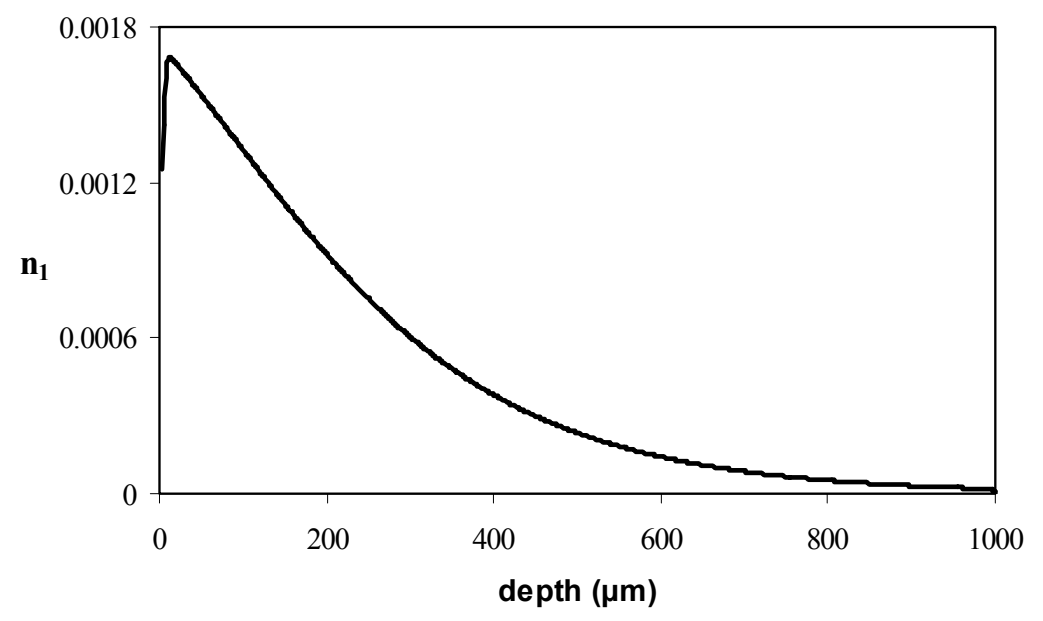

Figure 4. Simulation of the refractive index modulation as a function of the thickness.

Using these data and the RCW theory we can simulate the angular scan and check the theoretical results with an experimental layer of material based on PVA/AA. This comparison is done in figure 5, and the experimental results agree with the solutions obtained using the finite difference method. We can see the smoothing of the origins of the secondary lobes by the attenuation of the index profile $[19,21]$.

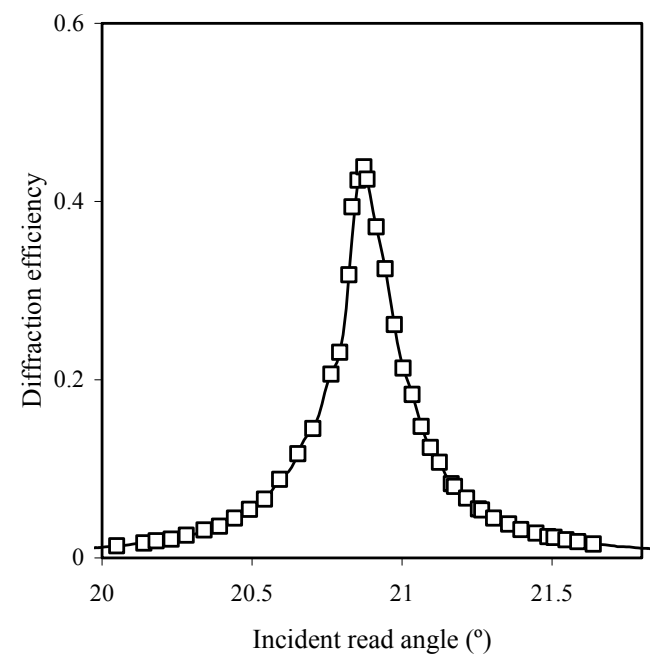

Figure 5. Simulation (continuous line) and experimental data (squares) of a $1000 \mu \mathrm{m}$ thick layer after $10 \mathrm{~s}$ of exposure.

\section{CONCLUSION}

We have used the finite difference method to solve the 3 D photopolymer behavior. We have analyzed the deviation of the sinusoidal shape in monomer and polymer gratings for different monomer diffusion values. We have evaluated the importance of the monomer diffusion in depth, and we conclude that it can be disregarded for short exposure times (around $10 \mathrm{~s}$ ). Finally we have obtained the refractive index modulation profile and checked the theoretical simulation with a polymer based on PVA/AA obtaining good agreement between theoretical simulation and experimental results.

\section{Acknowledgments}

This work was supported by the "Ministerio de Edicación y ciencia"(Spain) under projects FIS2005-05881-C02-01 and FIS2005-05881-C02-02 


\section{REFERENCES}

1. I. V. Kityk, J. Kasperczyk, B. Sahraouí, M. F. Yasinskii, and B. Holan, "Low temperature anomalies in polyvinyl alcohol photoplymers," Polymer 38, 4803-4806 (1997).

2. H. J. Coufal, D. Psaltis, Holographic Data Storage, G. T. Sincerbox, Springer-Verlag, New York, 2000.

3. S. Schultz, E. Glytsis, T. Gaylord, "Design, Fabrication, and Performance of Preferential-Order Volume Grating Waveguide Couplers," Appl. Opt. 39, 1223-1232 (2000).

4. D. A. Waldman, C. J. Butler, and D. H Raguin, "CROP holographic storage media for optical data storage at grater than 100 bits/ $\mu \mathrm{m} 2$, , in Organic Holographic Materials and Applications, 5316 (SPIE, San Diego, 2003).

5. W. L. Wilson et al., in Organic Holographic Materials and Applications, SPIE, San Diego, Vol. 5216, 178-179 (2003).

6. S. S. Orlov, W. Philips, E. Bjormson, Y. Takashima, P. Sundaram, L. Hesselink, R. Okas, D. Kwan, R. Snyder, "High-Transfer-Rate High-Capacity Holographic Disk Data-Storage System," Appl. Opt. 43, $4902-4914$ (2004).

7. R. R. McLeod, A. J. Daiber, M. E. McDonald, T. L. Robertson, T. S. Slagle, S. L. Sochava, and L. Hesselink, "Microholographic multilayer optical disk data storage," Appl. Opt. 44, 3197-3207, 2005.

8. M. Dubois, X. Shi, C. Erben, L. Longley, E. P. Boden, And B. L. Lawrence, "Characterization of microholograms recorded in a thermoplastics medium for three-dimensional optical data storage," Opt. Let. 30, 1947-1949, 2005.

9. Bablumian and T. Krile, "Multiplexed holograms in thick bacteriorhodopsin films for optical memory/ interconnections," Opt. Eng. 39, 2964-2974 (2000).

10. R. R. Adhami, D. J. Lanteigne and D. A. Gregory, "Photopolymer hologram formation theory", Microwave Optics Technology Letters 4, 106-109 (1991).

11. G. Zhao and P. Mouroulis, "Diffusion model of hologram formation in dry photopolymers materials," J. Mod. Opt. 41, 1929-1939 (1994).

12. J. Lougnot, P. Jost and L. Lavielle, "Polymers for holographic recording: VI. Some Basic ideas for modelling the Kinetics of the recording process," Pure and Appl. Opt. 6, 225-245 (1997).

13. S. Piazzolla y B. K. Jenkins, "First-harmonic diffusion model for holographic grating formation in photopolymers," J. Opt. Soc. Am. B 17, 1147-1157 (2000).

14. J. T. Sheridan and J. R. Lawrence, "Nonlocal-response diffusion model of holographic recording in photopolymer," J. Opt. Soc. Am. A 17, 1008-1014 (2000).

15. V. Moreau, Y. Renotte and Y. Lion, "Characterization of DuPont photopolymer: determination of kinetic parameters in a diffusion model," Appl. Opt. 41, 3427-3435 (2002).

16. R. L. Suhterland, V. P. Tondiglia, L. V. Natarajan, And T. J. Bunning, "Phenomenological model of anisotropic volume hologram formation in liquid-crystal-photopolymer mixtures," J. Appl. Phys. 2, 951-965 (2004).

17. S.-D. Wu, E. Glytsis, "Holographic grating formation in photopolymers: analysis and experimental results based on a nonlocal diffusion model and rigorous coupled-wave analysis," J. Opt. Soc. Am. B 20, 1177-1188 (2003).

18. J. V. Kelly, M. R. Gleeson, C. E. Close, F. T. O' Neill, J. T. Sheridan, S. Gallego and C. Neipp "Temporal analysis of grating formation in photopolymer using the nonlocal polymerization-driven diffusion model," Opt. Express , Vol: 13, 6990-7004 (2005).

19. S. Gallego, M. Ortuño, C. Neipp, A. Márquez, A. Beléndez, I. Pascual, J. V. Kelly, J. T. Sheridan, "Physical and effective optical thickness of holographic diffraction gratings recorded in photopolymers," Opt. Express 131939 (2005)

20. S. Gallego, M. Ortuño, C. Neipp, A. Márquez, A. Beléndez, I. Pascual, J. V. Kelly, J. T. Sheridan, "3 Dimensional analysis of holographic photopolymers based memories," Opt. Express 133543 (2005)

21. C. Neipp, J. T. Sheridan, S. Gallego, M. Ortuño, A. Márquez, I. Pascual and A. Beléndez, "Effect of a depth attenuated refractive index profile in the angular responses of the efficiency of higher orders in volume gratings recorded in a PVA/Acrylamide photopolymer" Opt. Comm. 233, 311-322 (2004).

22. C. Neipp, J. T. Sheridan, C. Pascual, A. Marquez, M. L. Álvarez, I. Pascual and A. Beléndez, "Finite difference time domain method (FDTD) to predict the efficiencies of the different orders inside a volume grating," in Photonic Engineering J. T. Sheridan, ed., Proc SPIE, Vol. 5827, Opto-Ireland ,Eire, (2005).

23. S. Blaya, L. Carretero, R.F Madriagal, M. Ulibarrena, P. Acebal, A. Fimia, "Photopolymerization model for holographic gratings formation in photopolymers," Appl. Phys. B 77, 639-662 (2003).

24. I. Aubrecht, M. Miler y I. Koudela, "Recording of holographic diffraction gratings in photopolymers: theoretical modelling and real-time monitoring of grating growth,” J. Mod. Opt. 45, 1465-1477 (1998). 
25. C. Neipp, A. Beléndez, S. Gallego, M. Ortuño, I. Pascual and J. T. Sheridan, "Angular responses of the first and second diffracted orders in transmission diffraction grating recorded on photopolymer material," Opt. Express 11, 1835-1843 (2003).

26. S. Gallego, C. Neipp, M. Ortuño, A. Márquez, A. Beléndez, I. Pascual "Diffusion based model to predict the conservation of holographic gratings recorded in PVA/Acrylamide photopolymer," Appl. Opt. 42, 5839-5845 (2003).

27. M. G. Moharam, E. B. Grann, D. A. Pommet and T. K. Gaylord, "Formulation for stable and efficient implementation of the rigorous coupled-wave analysis of binary gratings," J. Opt. Soc. Am. A 12, 1068-1076 (1995). 